Pires, A. M. L. T. "El prejuicio racial en Brasil: medidas comparativas"

\title{
EL PREJUICIO RACIAL EN BRASIL: MEDIDAS COMPARATIVAS
}

RACIAL PREJUDICE IN BRAZIL: COMPARATIVE MEASURES

\author{
Ana Maria Lacerda Teixeira Pires \\ Universidad Nacional de Educación a Distancia, Madrid, Espanha
}

\section{RESUMEN}

El estudio trataba de medir las manifestaciones del prejuicio racial en una muestra de la población brasileña, utilizando las escalas de racismo moderno de McConahay, Hardee y Batts (1981) y la escala de racismo cordial de Turra y Venturi (1995) y Venturi (2003). Se aplicó los cuestionarios a un total de 101 estudiantes de diversas universidades en la región sur de Brasil. Los resultados muestran que las dos escalas difieren entre si respecto a captar las expresiones del prejuicio. Las personas declararon mayor racismo moderno que racismo cordial. También hemos estudiado las variables que influyen en el prejuicio racial declarado por los participantes. La variable género y orientación a la dominancia social son variables que predicen el racismo moderno en la muestra estudiada. Los participantes indicaron, en mayor medida, la manifestación del prejuicio racial de forma encubierta y lo hacen más abiertamente cuando hay la posibilidad del contacto personal y estrecho.

Palabras clave: racismo moderno; racismo cordial; prejuicio.

\begin{abstract}
This study measured the manifestations of racial prejudice in a sample of Brazilian population, using McConahay, Hardees and Batts's scales of modern racism (1981) and Datafolha (1995) and Ventury, G's (2003) scale of cordial racism. The questionnaires were applied to a total of 101 students of several universities in southern Brazil. The results demonstrate that both scales differ among themselves according to capturing prejudice expressions. The citizens had declared greater modern racism than cordial racism. Also, the variables related with the influence on racial prejudice, stated by the participants, were analyzed. The gender and orientation to social dominancy variables are variables that predict the modern racism in the sample studied. The participants indicated, in greater measure, the manifestation of racial prejudice in an uncovered form, being this manifestation in a more open way, when there was the possibility of a more personal and closer contact.
\end{abstract}

Keywords: Modern racism; cordial racism; prejudice.

O PRECONCEITO RACIAL NO BRASIL: MEDIDAS COMPARATIVAS

\section{RESUMO}

O estudo avaliou as manifestações de preconceito racial em uma amostra da população brasileira, usando escalas de racismo moderno de McConahay, Hardee e Batts (1981), escala racismo cordial do Datafolha (1995) e Venturi, G. (2003). Os questionários foram aplicados a um total de 101 estudantes de várias universidades da região sul do Brasil. Os resultados mostram que as duas escalas diferem entre si sobre como capturar as expressões de preconceito. As pessoas relataram mais racismo moderno que racismo cordial. Estudamos também as variáveis que influenciam o preconceito racial declarado pelos participantes. As variáveis gênero e orientação à dominância social são preditores do racismo moderno na amostra estudada. Os participantes indicaram, em maior medida, a manifestação do preconceito racial dissimulado e o fazem mais abertamente quando existe a possibilidade do contato pessoal e estreito.

Palavras-chave: racismo moderno; racismo cordial; o preconceito.

\section{Introducción}

Muchos aspectos del racismo se reflejan, hoy en día, en la mayoría, de forma tácita, no demostrando así, actitudes abiertamente prejuiciosas con relación al objeto del prejuicio. Aunque se observe la tendencia abundante de las opiniones antirracistas y pese a que en las sociedades se sancione la discriminación por 
diferencias étnicas, se puede observar, sin embargo, que el racismo marca lo cotidiano y marca su presencia de distintas formas, incluso en las diferencias económicas y de bienestar social entre etnias o hasta autóctonos e inmigrantes. Los estudios relacionados con los afroamericanos en los Estados Unidos y con los inmigrantes en Europa nos ofrecen una vasta bibliografia al respecto.

De un modo general, podemos decir que los autores están de acuerdo en que las formas de expresar el racismo han cambiado y se han adaptado al control social, pero el racismo sigue existiendo en la mayoría. La ambivalencia de las actitudes racistas han generado diferentes conceptos. Así, Kinder \& Sears (1981) hablan del racismo simbólico, McConahay \& Hough (1976) del racismo moderno, Dutton (1976), Taguieff $(1987,1992)$ se refieren al racismo diferencialista, Meertens y Pettigrew (1993), Pettigrew y Meertens (1995) hablan del prejuicio sutil, Pérez, Falomir, Báguena y Mugny (1993) del racismo latente y Gaertner \& Dovidio (1986), hablan del racismo aversivo. Un aspecto común entre estos autores, es el de que la manifestación de este nuevo prejuicio tiene una naturaleza ambigua o contradictoria, y que el prejuicio se expresa en la actualidad de manera más indirecta, encubierta y sutil. Para McConahay (1986), esta diferencia se relaciona con el hecho de que el racista moderno se caracteriza, por ejemplo, por no aceptar las políticas consistentes en proporcionar ventajas a miembros de la mayoría desfavorecida con el fin de promover la igualdad (políticas de acción afirmativa, cuotas, etc), pero tampoco asume los estereotipos negativos tradicionales en contra los grupos objetos del prejuicio. Se entiende, por tanto, que las actitudes racistas ya no se expresan en términos de inferioridad del "otro" y en sentimientos segregacionistas, sino en términos de símbolos ideológicos abstractos y de comportamientos simbólicos donde prima el sentimiento de que el "otro" esta violando valores apreciados, lo que está demandando cambios en el status de su grupo que no están justificados. Otro aspecto de la ambivalencia del prejuicio, proviene segundo Katz y Hass (1988) del conflicto entre valores de igualitarismo y individualismo que, dependiendo del contexto, pueden traducirse en actitudes positivas o negativas hacia los miembros del exogrupo. Se trata de manifestaciones que, de vez en cuando, pasan desapercibidas para uno mismo, no dañando su autoimagen igualitaria y democrática socialmente valorada como positiva.

Dentro de las ciencias sociales, el origen del racismo es una combinación de factores psicológicos y culturales. A nivel psicológico, funciona como un mecanismo perceptivo de categorización (Allport, 1954) que agrupa y segmenta a las personas. El mecanismo afetivo, opera sobre el significado psicológico, y se refiere, al aspecto negativo que parece tener para la persona, todo aquello que sea extraño (por pertenecer a otra categoría) y, el significado positivo que parece tener, identificarse con algo similar o familiar, de su propia categoría.

De acuerdo con la Teoría de la Identidad Social (Tajfel \& Turner, 1979), el racismo como modalidad de relación entre grupos, combina el proceso de categorización social con la motivación a construir o mantener una identidad social positiva. Así, el racismo sería una modalidad de relaciones entre grupos como otras tantas, con mayor o menor intensidad según momentos y contextos específicos.

Wetherell y Potter (1992) proponen una explicación psicosocial basada entre el discurso racista, el contexto sociohistórico y en el análisis de ambos. Para estos autores, esto justifica, sostiene y legitima prácticas orientadas a mantener el poder y la dominación de unos grupos sobre otros. Por esta razón, las definiciones anteriores (racismo simbólico, sutil, moderno o aversivo) que caracterizan al racismo como un conjunto de pensamientos y sentimientos negativos sobre grupos, no pueden explicar el carácter cambiante del discurso racista o recurriendo a sus principios progresistas para justificar la exclusión de ciertos grupos. Desde esta perspectiva, el racismo es la expresión de ciertas relaciones de poder cuyas causas se sitúan en una estructura social de dominación, por parte de ciertos grupos y de opresión de otros grupos.

La teoria de la Dominancia Social conceptualizada por Sidanius y Pratto (1999) constituye un intento por reunir las ideas y aportaciones principales de las teorías que intentaban explicar la naturaleza humana de la opresión. La principal de sus descripciones básicas es que todas las sociedades humanas tienden a estructurarse como un sistema de jerarquías sociales basadas en los grupos. Esto quiere decir que uno o un reducido número de grupos dominantes o hegemónicos, estarán en lo más alto de la pirámide estructural, mientras los grupos subordinados estarán en la base. Además, el grupo dominante poseerá en gran medida los valores sociales positivos, esto es, las cosas materiales y simbólicas que las personas se esfuerzan en conseguir, como por ejemplo: el poder, alto estatus, bienestar, etc. Por el contrario, los grupos subordinados tendrán una amplia y desproporcionada cantidad de valores sociales negativos, tales como: bajo poder y estatus, ocupaciones con alto riesgo y de bajo estatus, etc. Los estudios sobre la orientación de dominancia social (véase Pratto et al., 1994) parten de la constatación que el conflicto entre grupos es una dimensión inevitable de la vida social. La teoría de la dominancia social intenta identificar los mecanismos que producen y mantienen esta jerarquía basada en los grupos sociales y cómo estos mecanismos interaccionan entre sí. Sidanius y Pratto (1999) distinguen entre los que ellos llaman jerarquías sociales basadas en los grupos, y 
las basadas en los individuos. Cuando hablan de jerarquía social basada en el grupo, ellos se refieren al poder social, prestigio y privilegio que un individuo posee por pertenecer a un determinado grupo social, como los derivados de pertenecer a una determinada raza, religión, clan, género, grupo étnico, linguístico, o clase social. En cambio, en las jerarquías sociales basadas en los individuos, éstos pueden disfrutar del poder, prestigio, etc. debido a sus características individuales (habilidades de liderazgo o atléticas, inteligencia, talento artístico, político, científico, etc). Estos mismos autores, distinguen tres sistemas de estratificación en las sociedades: uno basado en la edad, otro en el género, y el tercero, al que denominan sistema de divisiones arbitrárias, basado en características como la raza, etnia, estado, nación, secta religiosa, casta, clase social, grupo regional, o cualquier otra distinción grupal social relevante. De esta manera, señalan las tres admisiones básicas de la teoría de la dominancia social, es decir:

(a) Mientras que las jerarquías basadas en la edad y el género tenderán a existir dentro de todos los sistemas sociales, las jerarquías sociales de sistemas de divisiones arbitrarias surgirán siempre dentro de sistemas sociales que produzcan superávit económico;

(b) La mayoría de las formas de conflictos grupales y de opresión (racismo, etnocentrismo, sexismo, nacionalismo, clasicismo, regionalismo, etc) son diferentes manifestaciones de la misma predisposición humana básica para formar jerarquías sociales basadas en los grupos. Por tanto, la teoría de la dominancia social se centra en la forma en que ambos, el discurso social (ideología, actitudes y estereotipos) y las conductas individuales e institucionales, contribuyen y, a la vez son influidas por la naturaleza y severidad de las jerarquías sociales basadas en los grupos.

(c) Los sistemas sociales humanos sufren la tensión entre las fuerzas que intentan incrementar las jerarquías, tratando de producir y mantener altos niveles de desigualdad social basada en los grupos, y las fuerzas que intentan reducir las jerarquías, produciendo mayor igualdad social. La teoría argumenta que la jerarquía social basada en los grupos se produce y mantiene gracias a tres procesos: el conjunto de discriminaciones individuales, el de discriminaciones institucionales y la simetría conductual. Además, proponen que estos procesos se regularán por los mitos legitimadores que servirán a los individuos para apoyar un sistema de jerarquía social basado en los grupos. La orientación de dominancia social (SDO) consiste en la tendencia a preferir relaciones jerárquicas entre los grupos y a desear que el propio grupo de pertenencia sea superior a los otros grupos. Es uno de los factores psicológicos que inducen a la aceptación de los mitos de legitimación de la desigualdad (véase Sidanius \& Pratto, 1999).
En sus estudios Sidanius y Pratto (1999) han examinado la diferencia entre hombres y mujeres en la orientación hacia la dominancia social en 45 muestras independientes, con una muestra de casi 19.000 participantes en 10 países: Australia, Canadá, Israel, México, Palestina, China, Nueva Zelanda, Rusia, Suecia, y los EEUU. Los hombres tuvieron significativamente medias más elevadas en 39 de las 45 muestras, y no hubo ningún caso donde las mujeres tuvieron significativamente mayores niveles de de dominancia social que los hombres.

La contextualización histórica del racismo propuesta por Duckitt (1992) nos da una idea de que las definiciones del prejuicio y del racismo reflejan el entorno social y histórico de dichas relaciones raciales. Así la naturaleza y las formas de manifestación del prejuicio se encuentran bajo la influencia y normas sociales de un determinado entorno social. Esta nueva tipologia del racismo ha sido intensamente investigada en distintos contextos, como es el caso de los Estados Unidos y Europa. Ambos contextos tienen sus similitudes, diferencias y peculiaridades, y llegados aquí, desearíamos centrarnos en la contextualización del prejuicio racial en Brasil.

Los estudios llevados a cabo sobre el prejuicio racial, en los años 50, destacaron la distancia social existente entre blancos y negros, medidos por la "escala de distancia social de Bogardus" (Guimarães, 1999, p. 90). Estas investigaciones han demostrado los problemas de interacción entre estos grupos además de las dificultades de ascenso social experimentadas por los negros y mestizos en distintas regiones del país. Los resultados mostraron que los entrevistados, no dudaban en afirmar abiertamente sus prejuicios y evidenciaban el rechazo a una mayor posibilidad de proximidad social (Bastide, 1956; Bastide \& Van Den Berghe, 1957). Para varios autores, los resultados de estas investigaciones hacían patente la discrepancia entre la adhesión a los valores de igualdad, a las normas democráticas, la aceptación de la igualdad de oportunidades para blancos y negros, esto por un lado, y por otro, el alto grado de estereotipos negativos y la segregación en las relaciones personales por parte de los blancos con relación a los negros y mestizos. En los años sesenta, Bastide \& Fernandes trataron de estudiar las desigualdades raciales existentes en la sociedad brasileña. La importancia de los resultados de estas investigaciones, se caracterizaron, sobretodo, por contrastar con las ideas de que se consideraba el proceso histórico de mezcla racial en Brasil como un legado positivo, el que ha contribuido a la armonía de las relaciones raciales existentes y, de la misma manera, porque han destacado las diferencias sociales entre estos dos grupos. Así la ideologia de la "democracia racial", defendida por Freyre (1933), ha sido criticada y definida como "mito" al haber sido contrastada con 
las desigualdades sociales existentes en la sociedad brasileña (Bastide \& Fernandes, 1959).

Autores más recientes como Maggie y Gonçalves (1995) reconocen que en el presente se observa un sistema de relaciones raciales que colaboran a la división y clasificación de los grupos de acuerdo con sus diferencias, cuestionando el mito de la democracia racial como responsable por la negación del racismo.

En sus estudios más recientes, Turra y Venturi (1995), describen un tipo de racismo tipicamente brasileño, lo que llaman "racismo cordial". El racismo cordial, lo definen como una forma de discriminación en contra de los ciudadanos no blancos (negros y mestizos), que se caracteriza por una cortesía superficial que encubre actitudes y comportamientos discriminatorios y que se expresa en las relaciones interpersonales a través de chistes, dichos populares y bromas de carácter racial (véase Turra \& Venturi, 1995). Los resultados de la investigación de Turra y Venturi (1995), estan basados en un estudio que fue llevado a cabo en todo el territorio nacional y arrojó que el $89 \%$ de los brasileños reconocía la existencia del prejuicio racial. Aunque la mayoría de los sujetos admita que la sociedad brasileña es prejuiciosa, solo el $10 \%$ se ha considerado personalmente prejuicioso.

Estos autores han utilizado una escala menos directa para medir el "racismo cordial" y han verificado que más del $50 \%$ de los entrevistados estaban de acuerdo con las afirmaciones de dicha escala y un $83 \%$ de los sujetos declararon estar de acuerdo con algún aspecto de los ítems de la escala de racismo cordial (véase Turra \& Venturi, 1995).

En otro estudio, Martinez y Camino (2000), encontraron en esta misma línea, que los estudiantes universitarios cuando fueron preguntados por su grado de prejuicio en una escala del 1 al 10 (mayores puntuaciones indicaban mayor atribución de prejuicio) se atribuyeron a "si mismos" una media de 3,3 puntos, mientras que atribuyeron 7,8 puntos a "los brasileños en general".

Como puede observarse en los estudios revisados, en el contexto brasileño, la mayoría de los entrevistados no detecta en sí mismo una conciencia de ser racista, al menos demuestran voluntariamente su conducta de no serlo (yo no soy racista, eso son los otros). Por otro lado, la creciente preocupación de la sociedad con respecto a las desigualdades sociales y el mayor número de estudios mejor documentados que se desarrollaron en las últimas décadas no sólo no corroboran las ideas optimistas acerca de las relaciones raciales en Brasil (Fernandes, 1972; Nascimento, 1968) como también confirman las desigualdades raciales y asocian la idea del racismo a las oportunidades desiguales entre los grupos.

Los datos de la Síntese de Indicadores Sociais 2005 elaborada por el IBGE - Instituto Brasileiro de Geografia e Estatística (2005), a partir de la Encuesta Nacional por Amostra de Domicílios de 2004, han demostrado las diferencias entre la población blanca y la población afrodescendiente en lo que se refiere al mercado de trabajo y la formación escolar. Según estos resultados el salario de los trabajadores negros en Brasil es, en promedio, $50 \%$ menor que el de los blancos. La desigualdad racial se manifiesta también con relación a la asistencia a la escuela. Los blancos poseen una media de 9,8 años de estudio, lo que representa 2,1 años de estudio más que la población afrodecendiente.

Por lo tanto y resumiendo, las investigaciones realizadas no ofrecen dudas acerca de la percepción del prejuicio racial por parte de la mayoría de los encuestados $\mathrm{y}$, como ya hemos reseñado anteriormente los indicadores sociales de desigualdades ponen de manifiesto las diferencias entre los ciudadanos blancos y no blancos.

Nuestra intención, llegados a este punto, es estudiar las manifestaciones del prejuicio racial, medidas a través de las Escalas de Racismo Moderno de McConahay, Hardee y Batts (1981) y la Escala de Racismo Cordial, Turra y Venturi (1995) y Venturi (2003) en una muestra de la población brasileña.

\section{Definición operativa de afrodescendiente}

En Brasil los afrodescendientes corresponden a la suma de "pretos" (negros) y "pardos" (mestizos).

Tabla 1 - Distribución de la población : cor o raza en porcentuales (\%)

\begin{tabular}{|l|c|c|c|c|}
\hline & Blanca & Negra & Parda & Amarilla / Indígena** \\
\hline Brasil & 54,0 & 5,4 & 39,9 & 0,6 \\
\hline Región Norte* & 28,4 & 2,3 & 68,63 & 1,0 \\
\hline Región Noreste & 29,7 & 5,6 & 64,5 & 0,2 \\
\hline Región Sureste & 64,0 & 6,7 & 28,4 & 0,8 \\
\hline Región Sur & 83,6 & 3,0 & 12,6 & 0,7 \\
\hline Región Centro-Oeste & 46,2 & 3,5 & 49,4 & 0,8 \\
\hline
\end{tabular}

Fuente: Encuesta nacional de muestras por hogares (IBGE,2000)

*Solo la población urbana **Las dos categorías van juntas. 
Debe resaltarse que la definición de lo que es "afrodescendiente", en su aspecto conceptual es algo sobre lo que no existe consenso y parece llevar tanto a sobreestimaciones como a subestimaciones de la cantidad de población de estos pueblos, dependiendo de los criterios empleados (auto definiciones o censos $\mathrm{y}$ encuestas oficiales).

La necesidad de evitar falsas identificaciones y de adaptarse a diferentes clasificaciones (relacionadas al color de la piel) ha hecho que, en este estudio se utilice el criterio básico de contraponer los afrodescendientes al resto de la población considerada blanca se utilize como sinónimos los términos "afrobrasileños - negros y mestizos".

\section{Objetivos de la presente investigación}

En las últimas décadas se han elaborado nuevos instrumentos "más sutiles" destinados a captar las nuevas formas de expresar el prejuicio. Con este propósito McConahay, Hardee y Batts (1981) han creado la Escala de Racismo Moderno como instrumento menos reactivo que las escalas convencionales para medir el prejuicio racial. En Brasil, se ha utilizado la Escala de Racismo Cordial para medir el prejuicio racial hacia los negros y mestizos (Turra \& Venturi, 1995; Venturi, 2003).

Por tanto, considerando el contexto que acabamos de describir, la presente investigación tuvo como objetivos: (a) Medir el prejuicio racial en una muestra de la población blanca utilizando dos instrumentos distintos y comparar ambos resultados; (b) Analizar la influencia de la orientación hacia la dominancia social sobre las dos medidas anteriores, a fin de verificar qué variables pueden estar influyendo en las posibles diferencias en el prejuicio declarado en dichas escalas.

\section{Método}

\subsection{Muestra}

En este estudio participaron 101 sujetos de la raza blanca (25 mujeres y 76 hombres; el $24,8 \%$ y el $75,2 \%$ respectivamente). Sus edades oscilan entre 17 y 50 años con na media de 23,36 (d.t. $=5,61$ ). Con referencia a la ocupación, el 65,3 \% de los sujetos solo estudia y el $34,7 \%$ estudia y trabaja.

\subsection{Procedimiento y Instrumento}

Los estudiantes que participaron en este estudio contestaron un cuestionario, en el que, además de indicar la raza de pertenencia, el sexo, edad y ocupación se incluían las seguintes secciones:

1- Una versión de la escala de racismo moderno de McConahay, Hardee y Batts (1981) compuesta de 10 ítems (como se cita en Navas, 1998).

2- La Escala de Racismo Cordial (Turra \& Venturi, 1995; Venturi, 2003) compuesta de 12 ítems.

3- La escala de Orientación a la Dominancia Social SDO (Pratto et al., 1994). Esta escala, en la versión castellana traducida por Montes Berges y Silva Ferrero (como se cita en Moya \& Morales-Marente, 2005) recoge 16 enunciados sobre creencias acerca de las relaciones entre grupos. Las tres escala tienen un formato de respuesta tipo Likert, de 7 puntos (1 - totalmente en desacuerdo hasta 7 - totalmente de acuerdo). Las personas participantes en la investigación se contactaron en cuatro Universidades de la Provincia de "Rio Grande del Sur". Todos los sujetos participaran voluntariamente de la investigación y respondieran los cuestionarios de forma individual y anónima.

\section{Resultados}

\subsection{Estadisticos descriptivos y diferencia de medias entre prejuicio percibido y discriminación personal recibida}

En primer lugar, con el fin de analizar la fiabilidad de las escalas realizamos análisis de fiabilidad Alfa de Cronbach $(\alpha)$, de cada una de ellas. Los coeficiente obtenidos fueron de .68 para la escala de racismo moderno; .81 para la escala de racismo cordial y .84 para la escala de orientación a la dominancia social. Los resultados indican que las escalas muestran una fiabilidad adecuada. En la tabla 2 se presentan la fiabilidad y los estos estadísticos descriptivos para las tres escalas.

Tabla 2 - Estadísticos descriptivos y fiabilidad de las escalas

\begin{tabular}{|l|c|c|c|}
\hline \multicolumn{1}{|c|}{$\mathrm{N}=101$} & Media & D.T. & Alpha \\
\hline Escala de Racismo Moderno & 3.81 & 1.08 & .68 \\
\hline Escala de de Racismo Cordial & 1.95 & 1.12 & .81 \\
\hline Escala de Orientación a la Dominancia Social-SDO & 2.45 & 1.02 & .84 \\
\hline
\end{tabular}

La escala de respuesta oscila entre 1 (totalmente en desacuerdo) y 7 (totalmente de acuerdo)

Como puede verse en la tabla 2, las puntuaciones obtenidas por los sujetos en la escala de racismo moder- no (puntuación cercana a 4 sobre un máximo de 7) casi alcanzan el punto medio de la escala (4). Con relación a 
la escala de racismo cordial, la media total fue de 1.95 y es bastante inferior a media teorica de la escala (4). El prejuicio declarado en la escala de racismo moderno es significativamente más alto que el prejuicio declarado en la escala de racismo cordial. Estos resultados muestran con claridad que las dos escalas difieren entre si respecto a captar las expresiones del prejuicio.

Con el fin de analizar la diferencia entre las escalas de racismo moderno y de racismo cordial, hicimos una prueba $\mathrm{T}$ de muestras relacionadas. Esta diferencia es significativa

t $(100)=14.23 ; \mathrm{p}<0001$. Así, los sujetos de la muestra puntúan más alto en la escala de racismo moderno y declaran menor prejuicio en la escala de racismo cordial. Estos resultados indican que la escala de racismo moderno ha captado las expresiones más sutiles, simbólicas e indirectas de la manifestación del prejuicio racial, comparación con al escala de racismo cordial, en la muestra estudiada. Asimismo, se observa, que los sujetos tienen un nivel de orientación a la dominancia social inferior a la media teórica de la escala (2.45 sobre un máximo de 7).

\subsection{Análisis Factorial}

\subsubsection{Análisis factorial de la Escala de Racismo Moderno}

Un análisis factorial (método componentes principales con rotación Varimax) realizado sobre las respuestas a las escalas de racismo moderno (McConahay, Hardee, \& Batts, 1981) resultó la identificación de 4 factores que en conjunto explicaban el 63,71\% de la varianza total de la escala. El primer factor, RECONOCIMIENTO, que justificó el 20,33\% de la varianza total, está formado por aquellos ítems que se relacionan a las manifestaciones más simbólicas e indirectas del prejuicio, es decir, son referentes a la percepción de que "los afrobrasileños no necesitan ayudas especiales" (.79), seguido de que pueden "superar el prejuicio sin apoyo" (.69) que "están mejor ahora que nunca han estado" (66) y "reciben demasiado respeto y consideración, respectivamente" (.54). El Alpha de Cronbach es .65.

El factor 2, PESO EN LA SOCIEDAD, explicó un $16,94 \%$ de la varianza, en este caso el Alpha de Cronbach es .62 y agrupa los ítems que corresponden a la percepción de que los afrobrasileños "han conseguido más de lo que se merecen" (.80), "demasiada influencia sobre las decisiones políticas" (84) y en consecuencia la puntuación en la variable "es comprensible que estén descontentes" (-.44) que tiene una relación negativa. El factor 3, NEGACIÓN DE LA DISCRIMINACIÓN, explicó el 13,90\% de la varianza y está compuesto por el ítem 5, que satura con un peso muy alto "la discriminación no es un problema en Brasil” (.82) que corresponde precisamente la negación del problema del prejuicio y del racismo, además del item 3 "es comprensible que estén descontentes" (-.61), que tiene una relación negativa. Finalmente el factor 4, EXIGENCIAS EXCESIVAS (items 9 y 4) él que justificó el 12,53\% de la varianza total, resulta difícil de interpretar.

Por tanto, son los participantes que puntúan más alto en racismo moderno los que no están de acuerdo con la adopción de políticas de apoyo a los afrobrasileños y por consecuencia son los que declaran que la discriminación racial no es un problema en el contexto del estudio. Estos resultados indican que la escala de racismo moderno ha captado las expresiones más sutiles, simbólicas e indirectas de la manifestación del prejuicio racial, en línea con las ideas de McConahay (1986).

\subsubsection{Análisis factorial de la Escala de Racismo Cordial}

A continuación, realizamos un otro análisis factorial (método de componentes principales con rotación Varimax) sobre las respuestas a la escala de Racismo Cordial (Venturi, 2003) que resultó 4 factores que en conjunto explicaban el 62,09\% de la varianza total de la escala. El factor 1, el que explicó $23,50 \%$ de la varianza total, es a nuestro juicio un factor que se relaciona con el CONTACTO Y RECHAZO. Los ítems con más peso son respectivamente "me molestaría si tuviera un jefe negro" (.86), "si tuviera familias negras como vecinos" (.83), o "si un hijo se uniera en matrimonio con una persona negra" (.80), además del ítem 17 "si Dios ha hecho razas distintas es para que éstas no se mezclen entre si (.50) $\mathrm{y}$ en consecuencia la puntuación en la variable "hay gente buena y mala independientemente de la raza y esto no tiene que ver con el color de la piel" (-.54), tiene una relación negativa. El Alpha de Cronbach es .81. El segundo factor explicó el $(18,58 \%)$ de la varianza total, está compuesto por los ítems que corresponden a las MANIFESTACIONES CONCRETAS DEL PREJUICIO Y DISCRIMINACIÓN RACIAL. Dichos ítems que tienen los pesos más altos (15 y 13) se refieren a que los negros "siempre hacen tonterías" (.75) y que, "solo tienen habilidades para la música y el desporte" (.70) respectivamente. Para este factor el Alpha de Cronbach es .73. Los factores 3 y 4 , explican el 10,82\% y $9,18 \%$ de varianza total respectivamente. En el factor 3 , saturan solo 2 ítems que a nuestro juicio, son referentes a los PRINCIPIOS DE IGUALDAD Y DE JUSTICIA. Son ellos: item 12 "una buena característica del pueblo brasileño es el crisol de razas" (.69), y el item 16 "si pudieran tener una buena alimentación y estudiar, los negros tendrían éxito en cualquiera profesión"

(-.78) que tiene una relación negativa. Igual que en el factor 4, los ítems 14 "hay gente buena y mala independientemente de la raza, y esto no tiene que ver 
con el color de la piel" (.30) y el item 22 "la intención de votar en un político negro" (.89), obedecen a los principios de igualdad y justicia social.

En este caso, los que puntúan más alto en racismo cordial, son los que declaran mayor rechazo en el contacto con los negros y mestizos y los que evitan en mayor medida este contacto. Una otra conclusión de que se puede extraer de estos resultados es que independiente de la medida utilizada para medir el prejuicio racial, los participantes indican, por un lado, que la discriminación no es un problema en este contexto social y por tanto no apoyan las politicas favorables a los afrobrasileños, apoyando la teoria del racismo moderno. Por otro lado, cuando medimos el grado de contacto, los participantes expresan un alto grado de rechazo hacia este mismo grupo y expresan con claridad el prejuicio racial. Convenie señalar, que en el último caso, los resultados pueden estar sendo influenciados por la formulación de los ítems relacionados al prejuicio racial. Recordemos que los ítems que componen la escala de racismo cordial, miden el racismo manifiesto. A pesar de las diferencias, los resultados siguen a nuestro juicio, una pauta similar, lo que cambia es la manera de expresar el prejuicio racial, de acuerdo con el instrumento utilizado.

\subsubsection{Análisis factorial de la Escala de Dominancia Social}

Realizamos un análisis factorial sobre las respuestas a la Escala de Dominancia Social-SDO (Pratto at al., 1994) utilizando el mismo método anterior. Tras este análisis, obtuvimos que los ítems se agrupaban en cinco factores que en conjunto explicaban el $65,74 \%$ de la varianza total de la escala. Encontramos la fiabilidad para los cinco factores. El factor 1 explicó el 17,84\% de la varianza y teniendo en cuenta los ítems con los pesos más altos $(35,32,37,31$ y 30$)$ además del ítem 38 que tiene una relación negativa, podríamos considerarlo un factor que agrupa ítems referidos a promover IGUALDAD DE OPORTUNIDADES. La fiabilidad de este factor es alta (Alpha de Cronbach $=.81$ ).

El segundo factor, BÚSQUEDA DE JUSTICIA SOCIAL, él que explicó el $16,10 \%$ de la varianza total ( $\alpha$ de Cronbach $=.80$ ), saturan los items 27, 24 y 29 con pesos más altos.

El factor 3, DOMINANCIA SOCIAL (Alpha de Cronbach $=.77$ ), justificó el $12,45 \%$ de la varianza y está formado por los ítems que se relacionan a las manifestaciones claras y concordantes al dominio social de unos grupos sobre otros, (ítem 34 con un alto peso, seguidos de 33, 23 y 38 respectivamente). Los ítems 37,29 y 30 presentan valores negativos bajos y se mezclan con los de un factor diferente. El factor 4, POSICIÓN DE LOS GRUPOS EN LA SOCIEDAD, que justicó el 9,84\% de la varianza total, ( $\alpha$ de Cronbach .62), en él saturan los ítems 26 y 36 con un peso alto. Finalmente el factor 5 , que explicó el 9,49\% de la varianza y teniendo en cuenta los ítems con los pesos más altos ( 25 y 23 respectivamente) podríamos considerarlo como un factor de PREDOMINACIÓN DE UNOS GRUPOS SOBRE OTROS. Resulta llamativo observar que los participantes del estudio, apoyan la igualdad entre los grupos y la búsqueda de justicia social, pero hay un cierto número de participantes que apoya la predominación de unos grupos sobre otros.

\subsection{Correlación entre las escalas utilizadas en el estudio}

Tabla 3 - Correlaciones entre las escalas

\begin{tabular}{|l|c|c|c|}
\hline & Racismo Moderno & Racismo Cordial & Dominancia Social \\
\hline Racismo Moderno & - & $.29^{* *}$ & $.21^{*}$ \\
\hline Racismo Cordial & $.29^{* *}$ & - & $.37^{* *}$ \\
\hline Dominancia Social & $.21^{*}$ & $.37^{* *}$ & - \\
\hline
\end{tabular}

$* \mathrm{p}<.05 ; * * \mathrm{p}<.01$

Los resultados del análisis de correlación muestran una relación no muy alta, pero bastante significativa entre las escalas de racismo moderno y la de racismo cordial $(\mathrm{r}=.29 ; \mathrm{p}=0,003)$. En relación a la dominancia social y racismo moderno, observamos como existen relaciones bajas, aunque positivas $(\mathrm{r}=.21 ; \mathrm{p}<.05)$.

Por último, encontramos una relación positiva y elevada entre la dominancia social y el racismo cordial $(r=.37 ; p=0,000)$. De este modo, son las personas que presentan mayor tendencia a la dominancia social, las que puntúan más alto en racismo cordial.

\section{4 Análisis de Regresión}

\section{4. 1 Variables predictoras del prejuicio declarado en la escala de racismo moderno}

De acuerdo con el tercero objetivo de la investigación, realizamos un análisis de regresión para analizar la influencia de la orientación hacia la dominancia social sobre las escalas de racismo moderno y cordial con el objetivo de verificar qué variables pueden estar influyendo en las posibles diferencias en el prejuicio declarado en dichas escalas. 
En primer lugar tomamos como variable dependiente la puntuación de los sujetos en la escala de racismo moderno y como variable independiente la escala de dominacia social. Se incluyeron además las variables sexo, edad, ocupación, raza/color. Los resultados de la ecuación de regresión para racismo moderno pueden verse en la tabla 4.

Tabla 4 - Análisis de regresión para la variable dependiente Racismo Moderno

\begin{tabular}{|l|c|c|c|}
\hline & \multicolumn{3}{|c|}{ Racismo Moderno } \\
\hline Variables predictoras & Beta & $\mathrm{t}$ & $\mathrm{p}^{*}$ \\
\hline Orientación a la Dominancia Social & .213 & 2.235 & .028 \\
\hline Sexo & .254 & 2,669 & .009 \\
\hline \multicolumn{2}{|r|}{$\mathrm{R}^{2}=.112 ; \quad \mathrm{F}=6.183, \mathrm{p}(\mathrm{F})=.003$} \\
\hline
\end{tabular}

Como podemos ver en la tabla, la variable más importante es el sexo $(\beta=.25, \mathrm{p}=.009)$ seguida de orientación a la dominancia social $(\beta=.21, \mathrm{p}=.028)$ como variables predictoras del racismo moderno en los sujetos de la muestra estudiada. El modelo explica en su conjunto el $13 \%$ de la varianza total. $\left(\mathrm{R}^{2}=112\right.$; $(\mathrm{F}=6,18) ; \mathrm{p}<.001)$

\subsubsection{Variables predictoras del prejuicio} declarado en la escala de racismo cordial
Asimismo y con el objetivo de averiguar las posibles variables predictoras del grado de prejuicio declarado en la escala de racismo cordial por los sujetos de la muestra, volvimos a realizar un análisis de regresión, por el método de pasos sucesivos, utilizando como variable dependiente la puntuación de los sujetos en escala de racismo cordial y utilizando como variables predictoras la escala de orientación hacia la dominancia social. Se incluyeron también las variables sexo, edad, ocupación y raza. Se presentan en la tabla 5 los resultados de este análisis.

Tabla 5 - Análisis de regresión para la variable dependiente Racismo cordial

\begin{tabular}{|l|c|c|c|}
\hline & \multicolumn{3}{|c|}{ Racismo Cordial } \\
\hline Variables predictoras & Beta & $\mathrm{t}$ & $\mathrm{p}^{*}$ \\
\hline Orientación a la Dominancia Social & .372 & 3,982 & .000 \\
\hline & \multicolumn{2}{|c|}{$\mathrm{R}^{2}=.138 ; \mathrm{F}=15.858, \mathrm{p}(\mathrm{F})=.000$} \\
\hline
\end{tabular}

$* \mathrm{p}<.001$

Como puede verse en la tabla, la manifestación del prejuicio en la escala de racismo cordial es predicha por la variable orientación hacia la dominancia social $(\beta=.37$; $\mathrm{p}=.000$ ) en los sujetos de nuestro estudio. El modelo tiene significancia estadística alta y explica en su conjunto el $17 \%$ de la varianza total. $\left(\mathrm{R}^{2}=138 ;(\mathrm{F}=15,85) \mathrm{p}=.000\right)$.
Y por último, con el fin de analizar la diferencia entre la variable género y el prejuicio declarado en las escalas de racismo moderno y cordial, hicimos una prueba $\mathrm{T}$ de muestras independientes. Los resultados se puede observar en la tabla 6 .

Tabla 6 - Diferencias entre género y prejuicio declarado

\begin{tabular}{|l|l|l|c|c|c|}
\hline & \multicolumn{1}{|c|}{ Sexo } & N & Media & Desv. Típ & Sig* $^{* 1}$ \\
\hline Racismo Moderno & Mujer & 25 & 3.33 & 1.103 & .009 \\
\hline Racismo Cordial & Hombre & 76 & 3.97 & 1.041 & .014 \\
\hline & Mujer & 25 & 1.82 & 1.129 & n.s \\
\hline
\end{tabular}

$* \mathrm{p}<.001$ 
El resultado muestra que hay una diferencia estadísticamente significativa $\mathrm{t}(99)=-2,662 ; \mathrm{p}=0,09$ entre género y el prejuicio declarado en la escala de racismo moderno. Los sujetos del sexo masculino declaran mayor prejuicio en comparación con las participantes mujeres $\mathrm{t}(39,05)=-2,58, \mathrm{p}=.014)$ en el estudio. Con relación a los resultados que dicen respeto al prejuicio declarado en la escala de racismo cordial no produjo resultados significativos.

\section{Discusión y conclusiones}

Las teorías recientes sobre el prejuicio racial resaltan la paradoja presente en las sociedades modernas, en las que, por un lado, las personas no se reconocen racistas y, por otro lado, las desigualdades sociales tienden a aumentar. Las "nuevas teorias del prejuicio", buscan explicar el fenómeno, proponiendo la existencia de dos tipos de prejuicio, el manifiesto (antiguo) y el "moderno", "simbólico" o "sutill. Así, la mayoría de las investigaciones realizadas en este sentido muestran una tendencia, en la actualidad, a sustituir la manifestación del prejuicio racial por una forma más indireta, encubierta, atendiendo a la deseabilidad social que proclama la igualdad y la no discriminación.

Uno de los principales objetivos de la presente investigación era medir el prejuicio racial en una región de Brasil con mayor número de habitantes de la población de raza blanca, utilizando las escalas de racismo moderno y racismo cordial y comparar ambos resultados.

Utilizando la adaptación de la escala de McConahay, Hardee y Batts (1981), encontramos que la manifestación del prejuicio moderno hacia los afrobrasileños es más alta, mientras que la manifestación del prejuicio racial es significativamente más baja, cuando utilizamos la escala de racismo cordial (Venturi, 2003). Hay una diferencia estadísticamente significativa entre estas dos medidas, $\mathrm{t}(100)=14.23 ; \mathrm{p}<0001$. Estos resultados indican que las dos escalas difieren entre si respecto a captar las expresiones del prejuicio. A nuestro juicio, la escala de racismo moderno ha captado las expresiones más sutiles, simbólicas e indirectas de la manifestación del prejuicio racial, en comparación con la escala de racismo cordial, en la muestra estudiada. Asimismo, se observa, que los sujetos tienen un nivel de orientación hacia la dominancia social inferior a la media teórica de la escala, aunque hay un cierto número de participantes que apoya la dominación de unos grupos sobre otros. Estos datos confirman los resultados encontrados, relacionados al posicionamiento de los participantes hacia los afrodescendientes, como se comentará a continuación.

Una especificidad del presente estudio se relaciona al hecho de que, utilizando una misma muestra y dependiendo del instrumento utilizado para medir el prejuicio racial, hemos podido observar la manifestación de las dos formas del prejuicio (antiguo y moderno). Así, los participantes han puntuado más alto en la escala de racismo moderno, cuando han sido preguntados sobre cuestiones políticas e ideológicas relacionadas al prejuicio y, como cabría esperar, tuvieron más baja puntuación en la escala de racismo cordial (los ítems trataban de medir el prejuicio manifiesto). Estos resultados van en línea, con lo que mantienen las nuevas teorías del prejuicio y apoyan la teoria de McConahay (1986). Según este autor, se entiende, que las actitudes racistas ya no se expresan en términos de inferioridad del otro y en sentimientos segregacionistas, sino en términos de símbolos ideológicos abstractos y de comportamientos simbólicos donde prima el sentimiento de que el otro esta violando valores apreciados, lo que está demandando cambios en el status de su grupo que no están justificados. Por tanto, de acuerdo con lo expuesto, cuando presentamos cuestiones que trataban de medir el prejuicio manifiesto, obtuvimos una puntuación significativamente más baja. No obstante, vale la pena señalar que, en general, el nivel de prejuicio declarado hacia los afrobrasileños es medio, acentuándose la manifestación del rechazo hacia los negros y mestizos cuando existe la posibilidad del contacto "intimo", direto y cercano.

Otro de los objetivos que perseguíamos con este estudio era analizar las variables que influyen en la manifestación del prejuicio declarado en las escalas de racismo moderno y cordial. Los resultados obtenidos ponen de manifiesto la importancia de la variable género y orientación a la dominancia social para discriminar entre las personas que declaran mayor o menor grado de prejuicio racial en las dos escalas utilizadas en el estudio. Si atendemos al análisis de regresión por pasos de todas las variables utilizadas, el género es el mayor predictor para diferenciar la manifestación del prejuicio racial entre los sujetos que declaran un mayor racismo moderno. No obstante, respecto a la importancia de esta variable y su influencia sobre la escala de racismo cordial, ésta no aparece como predictora.

Los resultados de los análisis referentes a las diferencias entre género y prejuicio declarado muestran que los participantes del sexo masculino puntúan más alto en la manifestación de prejuicio moderno. Asimismo, examinado la diferencia entre hombres y mujeres en la muestra, nuestros resultados van en la misma línea con los encontrados por Sidanius y Pratto (1999), esto es, los hombres participantes en nuestro estudio, presentan mayores niveles de orientación hacia la dominancia social (SDO) que las mujeres, lo que queda demostrado por la mayor puntuación en la manifestación del racismo.

Una última conclusión que se puede extraer de estos resultados, tomados en su conjunto, es que 
la ambivalencia que caracteriza las nuevas actitudes prejuiciosas ha quedado también demostrada en el presente estudio. De esta manera, aunque los participantes apoyen la igualdad de oportunidades y justicia para todos, creen que algunos grupos deben estar sometidos a otros, e indican también una manifestación del prejuicio racial en mayor medida de forma encubierta evitando hacerlo de forma abierta. Así lo hacen, cuando existe la posibilidad de contacto personal y estrecho.

Finalmente, los resultados encontrados son congruentes con los estudios resumidos por Brown (1998). De acuerdo con este autor, cuando se utilizan medidas más indirectas y menos reactivas para medir el prejuicio, los datos obtenidos conducen al cuestionamiento de si es que realmente existe una disminución del prejuicio hacia los miembros de otros grupos étnicos.

\section{Referencias}

Allport, G. W. (1954). The nature of prejudice. Reading, MA: Addison Wesley.

Bastide, R. (1956). Stereotypes et prejuge de couleur. Sociologia. Revista Didática e Cientifica. Escola Livre de Sociologia e Política, 18(2), 141-171.

Bastide, R. \& Fernandes, F. (1959). Brancos e negros em São Paulo: ensaio sociológico sobre aspectos da formação, manifestações atuais e efeitos do preconceito de cor na sociedade paulistana (Coleção Brasiliana, Vol. 305). São Paulo: Cia. Editora Nacional.

Bastide, R. \& Van Den Berghe, P. (1957). Stereotypes, norms, and interracial behavior in São Paulo, Brasil. American Sociological Review, 22, 689-694.

Brown, R. (1998). Prejuicio. Su Psicología Social. Madrid: Alianza.

Duckitt, J. (1992). A historical analysis and integrative framework. American Psychologist, 47, 1182-1193.

Dutton, D. G. (1976). Tokenism, reverse discrimination, and egalitarianism in interracial behaviour. Journal of Social Issues, 32, 93-108.

Fernandes, F. (1972). O negro no mundo dos brancos. São Paulo: Difel.

Freyre, G. (1933). Casa Grande e Senzala: formação da família brasileira sob o regime da economia patriarcal. Rio de Janeiro: Maia \& Schmidt.

Gaertner, S. L. \& Dovidio, J. F. (1986). The aversive form of racism. In J. F. Dovidio \& S. L. Gaertner (Eds.), Prejudice discrimination and racism (pp. 61-89). Orlando, Fl: Academic Press.

Guimarães, A. S. A. (1999). Racismo e anti-racismo no Brasil. São Paulo: Editora 34

Instituto Brasileiro de Geografia e Estatística (IBGE). (2005). Síntese de Indicadores Sociais 2005. Acesso em 28 de março, 2008, em http:/www.ibge.gov.br/home/presidencia/noticias/ noticia visualiza.php?id noticia $=580 \&$ id pagina $=1$.

Katz, I. \& Hass, R. G. (1988). Racial ambivalence and American value conflict: Correlacional and priming studies of dual cognitive structures. Journal of Personality and Social Psychology, 55, 893-905.
Kinder, D. R. \& Sears, D. O. (1981). Prejudice and politics: symbolic racism versus racial threats to good life. Journal of Personality and Social Psychology, 40, 414-431.

Maggie, Y. \& Gonçalves, M. A. (1995). Pessoas fora do lugar: a produção da diferença no Brasil. In M. A. Gonçalves \& G. Villas Boas (Orgs.), O Brasil na virada do século (pp. 165-176). Rio de Janeiro: Relume-Dumará.

Martinez, I. \& Camino, L. (2000). Brasil es Racista, Brasil no es Racista: El discurso social como determinante de las diferencias percibidas entre blancos, negros y morenos. Comunicação apresentada no VII Congresso de Psicologia Social da Espanha, Oviedo, Espanha.

McConahay, J. B. (1986). Modern racism, ambivalence and the modern racism scale. In J. F. Dovidio \& S. L. Gaertner (Orgs.), Prejudice, discrimination and racism (pp. 91-126). New York: Academic Press.

McConahay, J. B., Hardee, B. B., \& Batts, V. (1981). Has racism declined in America? It depends upon who is asking und what is asked. Journal of Conflict Resolution, 25, 563-579.

McConahay, J. B. \& Hough, J. C. (1976). Simbolic Racism. Journal of Social Issues, 32, 33-45.

Meertens, R. W. \& Pettigrew, T. F. (1993). Le racisme voilé: Dimensions et measure. In M. Wiewiorka (Ed.), Racisme et modernité París (pp. 109-126). París: La Découverte.

Moya, M. \& Morales-Marente, E. (2005). Reacciones psicopolíticas ante los ataques terroristas del 11 de marzo de 2004. Revista de Psicología Social, 20, 331-350.

Nascimento, A. (1968). O negro revoltado. Rio de Janeiro. Nova Fronteira.

Navas, M. S. (1998). Nuevos instrumentos de medida para el nuevo racismo. Revista de Psicología Social, 13(2), 233-239.

Pérez, J. A., Falomir, J. M., Baguena, M. J., \& Mugny, G. (1993, Junio). El racismo: actitudes manifiestas y latentes. Papeles del Psicólogo, 57, 45-50.

Pettigrew, T. F. \& Meertens, R.W. (1995). Subtle and blatant prejudice in Western Europe. European Journal of Social Psychology, 25, 57-75.

Pratto, J., Sidanius, J., Stallworth, L., \& Malle, B. (1994). Social dominance orientation: A personality variable predicting social and political attitudes. Journal of Personality and Social Psychology, 67, 741-763.

Sidanius, J. \& Pratto, F. (1999). Social dominance. An intergroup theory of social hierarchy and oppression. Nueva York: Cambridge University Press.

Taguieff, P. A. (1987). La force du préjugé, essai sur le racisme et ses doubles. París: La Découverte.

Taguieff, P. A. (1992). Face au racisme. París: La Découverte

Tajfel, H. \& Turner, J. C. (1979). An integrative theory of intergroup conflict. In W. G. Austin \& S. Worchel (Eds.), The social psychology of intergroup relations (pp. 33-47). Monterey, CA: Brooks/Cole.

Turra, C. \& Venturi, G. (Orgs.). (1995). Racismo cordial - a mais completa análise sobre preconceito de cor no Brasil. São Paulo: Ática.

Venturi, G. (2003). Discriminação racial e preconceito de cor no Brasil. São Paulo: Fundação Perseu Abramo / Rosa Luxemburg Stiftung.

Wetherell, M. \& Potter, J. (1992). Mapping the language of racism: Discourse and the legitimation of explotation. Hemel Hempstead: Harvester Wheatsheaf. 
Pires, A. M. L. T. "El prejuicio racial en Brasil: medidas comparativas"

Recebido em: 05/08/2008

$1^{\text {a }}$. Revisão em: 28/03/2009

$2^{\text {a }}$. Revisão em: 05/01/2010

Aceite final em: 13/01/2010

Ana Maria Lacerda Teixeira Pires é Doutoranda em Psicologia Social, Departamento de Psicologia Social y de las
Organizaciones/Facultad de Psicología, Universidad Nacional de Educación a Distancia - UNED, Madrid, Espanha.

Email: anamariapires@hotmail.com

\section{Como citar:}

Pires, A. M. L. T. (2010). El prejuicio racial en Brasil: medidas comparativas. Psicologia \& Sociedade, 22(1), 32-42. 